\title{
Interacting with the SOA-Based Internet of Things: Discovery, Query, Selection, and On-Demand Provisioning of Web Services
}

\author{
Dominique Guinard, Student Member, IEEE, Vlad Trifa, Student Member, IEEE, \\ Stamatis Karnouskos, Senior Member, IEEE, Patrik Spiess, Member, IEEE, and \\ Domnic Savio, Member, IEEE
}

\begin{abstract}
The increasing usage of smart embedded devices in business blurs the line between the virtual and real worlds. This creates new opportunities to build applications that better integrate real-time state of the physical world, and hence, provides enterprise services that are highly dynamic, more diverse, and efficient. Service-Oriented Architecture (SOA) approaches traditionally used to couple functionality of heavyweight corporate IT systems, are becoming applicable to embedded real-world devices, i.e., objects of the physical world that feature embedded processing and communication. In such infrastructures, composed of large numbers of networked, resource-limited devices, the discovery of services and on-demand provisioning of missing functionality is a significant challenge. We propose a process and a suitable system architecture that enables developers and business process designers to dynamically query, select, and use running instances of real-world services (i.e., services running on physical devices) or even deploy new ones on-demand, all in the context of composite, real-world business applications.
\end{abstract}

Index Terms-Service-Oriented Architecture (SOA), service discovery, web services, REST, Web of Things, device integration, composite applications, wireless sensor (actuator) networks, context modeling, ubiquitous business processes.

\section{INTRODUCTION}

\subsection{Motivation}

THE last years, we have witnessed two major trends in the world of embedded devices. First, hardware is becoming smaller, cheaper, and more powerful. According to the Internet of Things vision (IoT) [1], the majority of the devices will soon have communication and computation capabilities, which they will use to connect, interact, and cooperate with their surrounding environment. Second, the software industry is moving towards service-oriented integration technologies. Especially in the business software domain, complex applications based on the composition and collaboration among diverse services have been appearing. The Internet of Services vision (IoS) [2] assumes this on a large scale, where services reside in different layers of the enterprise, e.g., different operational units, IT networks, or even running directly on devices and machines within the company. As both of these trends are not domain specific but common to multiple industries, we are facing a trend where the service-based information systems blur the border between the physical and virtual worlds, providing a fertile ground for a new breed of real-world aware applications.

- D. Guinard and V. Trifa are with the SAP Research and Institute for Pervasive Computing, ETH Zurich, Kreuzplatz 20, 8008 Zurich, Switzerland. E-mail: \{dominique.guinard, mihai.vlad.trifa@sap.com.

- S. Karnouskos, P. Spiess, and D. Savio are with SAP Research Karlsruhe, Vincenz-Priessnitz-Strasse 1, D-76131 Karlsruhe, Germany.

E-mail: \{stamatis.karnouskos, patrik.spiess,domnic.savio\}@sap.com.

Manuscript received 12 June 2009; revised 15 Oct. 2009; accepted 20 Nov. 2009; published online 10 Feb. 2010.

For information on obtaining reprints of this article, please send e-mail to: tsc@computer.org, and reference IEEECS Log Number TSCSI-2009-06-0165. Digital Object Identifier no. 10.1109/TSC.2010.3.

1939-1374/10/\$26.00 (C) 2010 IEEE
The efficiency of such applications will heavily depend on the cooperation of heterogeneous networked embedded devices among themselves and with business systems [3]. In this Internet of Things, we expect that dynamic network discovery, query, selection, and on-demand provisioning of web services will be of crucial importance.

In the future Internet, real-world devices will be able to offer their functionality via SOAP-based web services (WS-*) or RESTful APIs [4], enabling other components to interact with them dynamically. The functionality offered by these devices (e.g., the provisioning of online sensor data) is often referred to as real-world services because they are provided by embedded systems that are related directly to the physical world. Unlike traditional enterprise services and applications, which are mainly virtual entities, real-world services provide real-time (we refer to relatively low-latency, not necessarily offering hard real-time guarantees) data about the physical world. Armed with this additional knowledge, one can support a more efficient decision taking process. Hence, devices providing their functionality as a web services can be used by other entities such as enterprise applications or even other devices. No device drivers are needed anymore and a new level of efficiency can be achieved as web service clients can be generated dynamically at runtime.

Trends show that in the future, a much more diversified infrastructure will emerge, and the way we interact with it will change accordingly. As depicted in Fig. 1, mash-ups of services will be created and used across various system layers. We will experience horizontal collaboration directly between devices with no human intervention [3], but also vertical collaboration between devices and online services, applications, and people. Enterprise applications will be able to connect directly to devices with no need for proprietary 


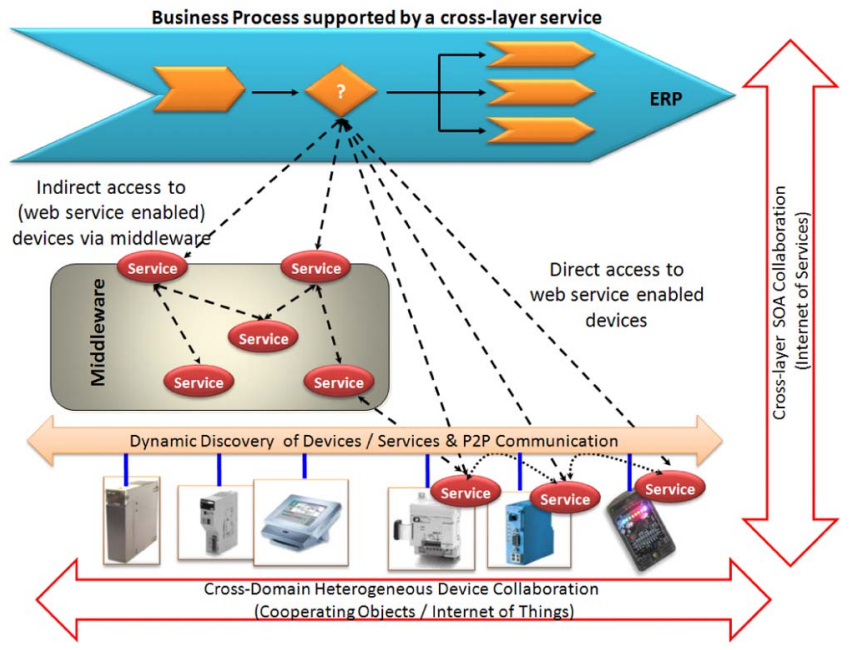

Fig. 1. The collaborative future Internet vision.

drivers, while non-web service-enabled devices can still be used by wrapping their functionality behind gateways. Peerto-peer communication among devices will push services down to the device layer and create new opportunities for functionality discovery and collaboration.

According to OnWorld [5], the global market for Wireless Sensor Network (WSN) systems and services is expected to skyrocket to about $\$ 4.6$ billion in 2011, up from approximately $\$ 500$ million in 2005 . There will be a worldwide market of $\$ 5.3$ billion (conservative estimate) for the industrial control segment only, comprising 4.1 million nodes by 2010. OnWorld's most aggressive forecast for all wireless sensor (\& control) network segments is $\$ 8.2$ billion by 2010, comprising 184 million deployed nodes. Thus, the business opportunities for real-world services are promising. Even if a fraction of this holds true for the near term, we will witness a mass market penetration of networked embedded devices. Services taking advantage of the unprecedented ease of consumption of device functionality will give birth to new innovative applications and provide both revenue generating and cost saving business advantages. From a technology point of view, the key challenge is how to discover, assess, and efficiently integrate the realworld services into business applications.

\subsection{Background and Related Work}

Several efforts have explored the integration of real-world and enterprise services, e.g., [6], [7]. However, the protocols used do not offer uniform interfaces across the application space and are too complex to integrate with traditional enterprise applications. To ensure interoperability across all systems, recent work has focused on applying the concept of Service-Oriented Architecture (SOA), in particular web service standards (SOAP, WSDL, etc.) directly on devices (e.g., [8], [9], [10]). Implementing WS-* standards on devices presents several advantages in terms of end-to-end integration and programmability, by reducing the need for gateways and translation between the components. This enables the direct orchestration of services running on devices, e.g., sensors monitoring the temperature of shipments, with high-level enterprise services, e.g., offered by an Enterprise Resource Planning (ERP) application.
Embedding SOA concepts at device level initially seems a good idea; however, we have to keep in mind that SOA standards were designed primarily for connecting, complex, and rather static enterprise services. Thus, implementing WS-* standards directly on devices is not always straightforward. Unlike enterprise services, real-world services are deployed on resource constrained devices, e.g., with limited computing, communication and storage capabilities. This requires significant simplification, optimization, and adaptation of SOA tools and standards [10]. Additionally, realworld services are found in highly dynamic environments where devices and their underlying services constantly degrade, vanish, and possibly re-appear. As such, this infrastructure can not be considered as static and long-lived as traditional enterprise services. This implies the need for automated, immediate (dynamic) discovery of devices, and services as well as their effective management.

A crucial challenge for SOA developers and process designers is to find adequate services for solving a particular task [11]. This process is often referred to as "service discovery" or simply "discovery" [12] and is enduser driven. We shall distinguish it from the "network discovery" [7] of services which is machine driven and occurs at the network level. Discovering enterprise services often implies manually querying a number of registries, such as Universal Description and Discovery and Integration (UDDI) registries, and the results depend largely on the quality of the data within that registry. While such an approach is adequate for a rarely changing set of large-scale services, the same is insufficient for the requirements of the dynamic real-world services. Registering a service with one or more UDDIs is rather complex and does not comply with the minimization of usage of the devices' limited resources. Furthermore, extensive description information is necessary [13] while the device can only report basic information about itself and the services it hosts. Trying to reduce the complexity of registration and discovery, different research directions have been followed in order to provide alternatives or extensions of the UDDI standard [11], [12], [14]. However, also these do not take into account the particular requirements of real-world services.

\subsection{Our Contributions}

Based on our experiences within SAP, in developing realworld services for the enterprises, we introduce here a set of requirements to facilitate the querying and discovery of real-world services from enterprise applications:

1. R1: Minimal Service Overhead. As most real-world services are offered by embedded devices with (very) limited computing capabilities there is a need for a lightweight service-oriented paradigm which does not generate too much overhead compared to using functionality through the proprietary APIs.

2. R2: Minimal Registration Effort. A device should be able to advertise its services to an open registry using network discovery. The process should be "plug and play", without requiring human intervention. A device should also be expected to provide only a small amount of information when registering.

3. R3: Support for Dynamic and Contextual Search. It should be possible to use external sources of 
information to better formulate queries. Furthermore, the queries should go beyond simple keyword search and take into account user-quality parameters such as context (e.g., location, Quality of Service (QoS), application context). Support for context is essential as the functionality of most real-world devices is task-specific within a well-defined context (e.g., a building, a manufacturing plant, etc.).

4. R4: Support for On-Demand Provisioning. Services on embedded devices offer rather atomic operations, such as obtaining data from a temperature sensor. Thus, while the WSN platforms are rather heterogeneous, the services that the sensor nodes can offer share significant similarities and could be (re)deployed on-demand per developer request.

In the work presented here we build upon existing research on device integration through services [8], [9], [10], [15]. Our key contribution is the service discovery process for real-world services initially introduced in [16]. This process is shown on Fig. 4 and described in detail in Section 4 . The goal of this process, called Real-World Service Discovery and Provisioning Process (RSDPP), is to assist the developers at development time in the discovery of realworld services to be included in composite applications. This innovative process fulfills the requirements of real-world services we described (R1 to R4) above as follows: In order to ensure a minimal overhead (R1) for providing the functionality of embedded devices as service we propose two approaches. In the first one we use the Device Profile for Web Services (DPWS) [8] and its dynamic network discovery mechanism. DPWS defines a limited set of WS-* standards which are implementable on relatively resource-constrained devices. We will describe DPWS in Section 3.1.

As an alternative to fulfill requirement $R 1$, we also introduce the design of Resource Oriented real-world devices that is embedded devices providing their functionality through a RESTful API [4], [17], [18]. REST (Representational State Transfer) [19] is the architectural principle that lies at the heart of the web and shares a similar goal with DPWS, which is to increase interoperability for a looser coupling between the parts of distributed applications [20] toward serendipitous reuse of services. We further describe the concept of resource-oriented real-world devices in Section 3.2.

The minimal registration effort (R2) requirement is met by using DPWS [8] and its network discovery mechanism. In Section 3.2, we also describe how Resource Oriented devices can also fulfill this requirement.

We further ensure the minimal registration (R2) effort and support for dynamic search (R3) by extending developer provided keywords with vocabularies of related terms also known as "lightweight ontologies" [21]. We generate these terms dynamically, by using semistructured web resources like Wikipedia and Yahoo! Web Search. This part of the process called Query Augmentation, is described in Section 4.1.

The dynamic search requirement (R3) is also fulfilled by taking into account the developer context and matching it with the extracted context of real-world services. This developer quality information is then used for adequate service selection when retrieving and ranking services as explained in Section 4.2.
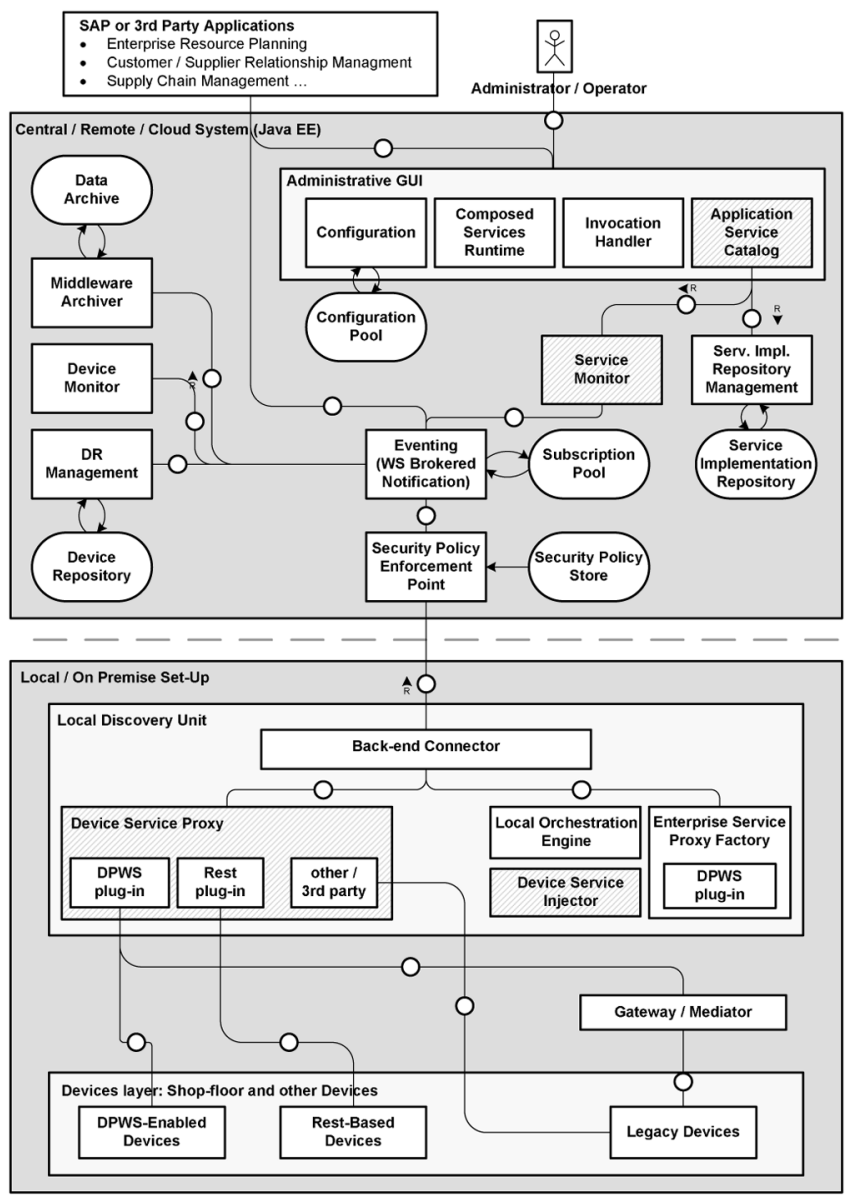

Fig. 2. The SOCRADES integration architecture (SIA).

The requirement for on-demand dynamic provisioning (R4) is fulfilled by a software architecture that enables the developer to automatically deploy services on devices when no requirements-satisfying service was found in the environment [22]. This architecture is described in Section 4.3.

Finally, we present our implementation within an enterprise application (based on Java Enterprise Edition and SAP NetWeaver) as well as its deployment and validation of our results in Section 5 .

Before describing the process itself, we start with an overview of the framework in which the RSDPP was developed and in particular on how devices can register themselves and advertise their services in an automated manner.

\section{The SOCRADES Integration Architecture}

The process described in this paper has been developed and implemented as part of the SOCRADES Integration Architecture (SIA) [9], [23], [24], which is depicted in Fig. 2. The role of SIA is to enable the ubiquitous integration of realworld services running on embedded devices with enterprise services. WS-* web service standards constitute the de facto communication method used by the components of enterprise-level applications, and for this reason SIA is fully based on them. In this manner, business applications can access near real-time data from a wide range of networked devices through a high-level, abstract interface based on 
web services. Furthermore, the SIA also supports RESTful services in order to be able to communicate with many emerging Web 2.0 services. This enables any networked device that is connected to the SIA to directly participate in business processes while neither requiring the process modeler, nor the process execution engine to know about the exact details of the underlying hardware.

The details of SIA can be found in [24]; therefore, here, we focus those components of the architecture, which are relevant for RSDPP. The components with gray, diagonal patterns play a key role in the process. SIA is split in two parts: A "local, on premise" part which features a Local Discovery Unit (LDU) and is running at the local network that contains the devices to be integrated, and a central system (anywhere on the network or even Internet) that hosts enterprise-level applications. Although the quantitative relation between both subsystems is $m: n$, in a typical single-site setup there will be only one central system and one or more on-premise systems. In a multienterprise collaborative landscape, however, we would witness several "central" systems collaborating and possibly various "local" systems reporting to more than one "central" ones.

In the local subsystem at Device Layer there are several embedded devices that are running various services. SIA is able to interact with devices using several communication protocols, such as DPWS, OPC-UA, REST, etc., however, in this paper, we focus solely on DPWS and REST-enabled devices. Since DPWS-enabled devices support web services, they also can bypass SIA for a direct connection to Enterprise Applications, which is desirable in some use cases, but not for the majority of foreseen ones. Furthermore, SIA allows applications to subscribe to any events sent by the devices, offering a publish/subscribe component that supports WS-Notifications. It also offers buffered invocations of hosted services on devices that are only intermittently connected, by receiving notifications when the device becomes available again or having the system cache the message and delivering it when the device is ready to receive it. As such SIA is a vital component hiding and managing the complexity of real-world landscapes from the enterprise service developers, easing their tasks.

The key component that connects the local subsystem with the central one is the local network discovery unit (or LDU). The LDU module Device Service Proxy scans the local network for DPWS and REST devices and reports their connecting and disconnecting to the central system. It acts as an intermediary that provides uniform access to different classes of devices through a set of platform-dependent plugins. Some of the advanced features of the LDU are a lightweight local orchestration engine that allows for autonomous execution of local processes, a Device Service Injector that is able to change the embedded software on devices in order to (un)deploy or (re)configure embedded services (used for on-demand provisioning as in Section 4.3.2), and an enterprise service proxy factory that can make services from a business application available in the local network so that devices can access those back-end services through the same protocols as they would use to communicate with other devices. All these are realized by (unidirectional) web service calls from the LDU to the central system, therefore, allowing for firewall-friendly operations and operation through an HTTP(s) proxy.
In the central subsystem, we have implemented higherlevel components to ease the management and use of devices in a standardized and uniform way. The Device Repository holds all dynamically acquired but mostly static device information (metadata) of all online and offline devices (of all connected local subsystems), while the Device Monitor contains information about the current state of each device. The Device Monitor acts as the single access point where enterprise applications can find all devices even when they have no direct access to the shop floor network or such access is not wished (e.g., flooding of shop-floor with network discovery messages).

At the same time, information about the different services hosted on the device (typically described using WSDLs) will be retrieved and forwarded using an event to the Service Type Repository and Service Monitor as shown on Fig. 4. The former only contains information about the service types without their respective endpoint references; the latter contains information about the available service instances hosted by the devices and their endpoint references, and also installable service types. The Service Type Repository acts as a facade for querying the underlying repositories and monitors for pointers to running service instances.

The RSDPP process is mainly orchestrated from the component called Application Service Catalog. It contains a GUI, hosts and applies the service instance ranking strategies (see Section 4.2.3), and controls the interaction with the other components involved in the process.

\section{Network Discovery of Embedded Devices}

Along with increasingly dynamic infrastructures where mobile devices appear or disappear from the network at operation time, there is a strong need for tools to simplify the management and interconnection of networked devices. Network discovery is a central process in ubiquitous and distributed computing [7]. In contrast to the user-oriented discovery, network discovery enables machines to automatically register themselves and advertise their services on the network. In a way, network discovery is the bootstrap of service discovery for end-users. In this field, many protocols have been proposed such as the Service Location Protocol (SLP), Universal Plug and Play (UPnP), Device Profile for Web Services, Sun's Jini, or Apple's Bonjour.

Such a discovery mechanism is essential in scenarios where devices can join the network and discover dynamically the services offered by other devices, and will be unavoidable requirement in future enterprise scenarios with dynamic and adaptive production lines. In this section, we present three alternative mechanisms we have used in our system for network discovery of devices. As depicted in Fig. 3, the process of finding out real world services running on physical devices can be done as follows:

1. WS-Discovery on which DPWS is based (both active and passive).

2. RESTful active network discovery, where a device notifies its presence to the LDU automatically.

3. Passive RESTful discovery for REST-enabled devices that do not comply with SIA network discovery. Passive RESTful discovery is triggered by passing the URI of the device to be registered in the system as a parameter of the forced discovery method call. 


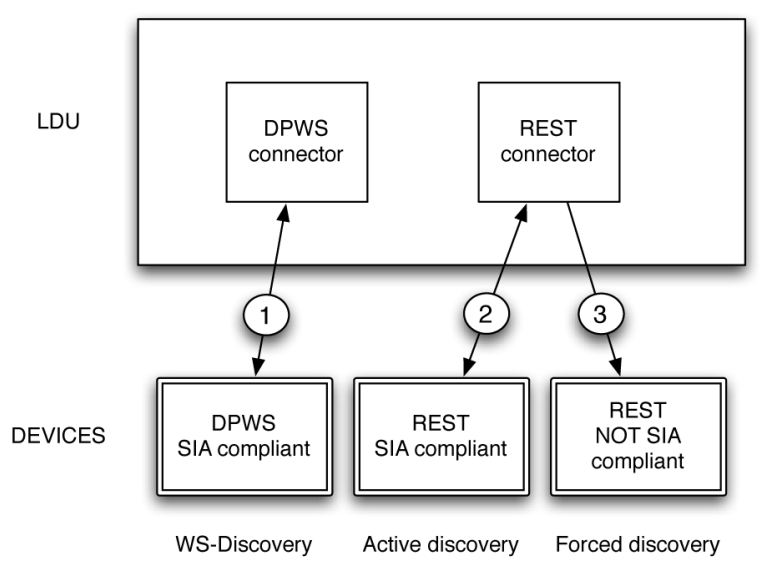

Fig. 3. Three alternative mechanisms for network discovery of real-world services hosted on physical devices.

\subsection{DPWS Web Services}

Device Profiles for Web Services (DPWS) is a subset of web service standards (such as WSDL and SOAP) that allows minimal interaction with web services running on embedded devices. DPWS is the successor of Universal Plug and Play (UPnP) as in essence it specifies a protocol for seamless interaction with the services offered by different embedded devices. However, DPWS is fully aligned with web services technologies. The various specifications DPWS include support for (secure) messaging, service discovery, and description, and eventing for resource-constrained devices. Devices can run two types of services: 1) hosting services and 2) hosted services. Hosting services are directly related to DPWS and support the low-level, generic (meta)services such as network discovery services used by a device connected to a network to advertise itself and to discover other devices, metadata exchange services to provide information about a device and the hosted services on it, and asynchronous publish and subscribe eventing, allowing to subscribe to asynchronous event messages produced by a given hosted service. Hosted services are mostly functional and depend on their hosting device and its functionality for network discovery.

As services run directly on limited networked devices, a robust mechanism is needed, in order to find new devices as they connect to the network, and dynamically retrieve metadata about it and the services it hosts. To achieve this, the WS-Discovery specification is used. When a new device joins the network, it will multicast a HELLO message via the UDP protocol. By listening to this message, clients can detect new devices and in a second step retrieve their metadata. This in turn triggers the sending of an appropriate message to the SIA Device Monitor, containing the device's static metadata. The metadata information can be classified into a certain set of metadata classes (see categories paragraph below), and is required for searching services according to more detailed criteria. This data about the device is stored by the higher units for future usage.

Filtering information can be included in a Probe message sent to a multicast group. Devices whose metadata match the probes' content will send a ProbeMatch response directly to the client (in unicast mode). Similarly, to locate a device by name, a client sends a Resolve message to the same multicast group and the device that matches sends a ResolveMatch response directly to the client. After this network-level scan, the result set can be further narrowed by matching keywords or textual information that describe both static (device type, available sensors on board) and dynamic properties of devices (QoS, physical location, available battery life, network connectivity, or available sensors).

Metadata categories. The DPWS metadata of devices and services can be classified in different categories, as follows:

- Scopes a set of attributes that may be used to organize devices into logical or hierarchical groups, e.g., according to their location or access rights.

- Model and Device metadata provides information about the type of the device like manufacturer name, model name, model number, etc. as well as information on the device itself such as serial number, firmware version, and friendly name.

- Types are a set of messages the device can send and/ or receive; these can be either functional WSDL port types (e.g., "turn on," "turn off") or abstract types grouping several port types and/or hosted services (e.g., "printer," "lighting," "residential gateway").

- Links to WSDL document (i.e., URLs), containing the port types (operations and message structures) implemented and the endpoint of hosted services.

\subsection{RESTful Services for the Real-World}

The architectural principles that are core to the web, namely Representational State Transfer (REST) as defined by Roy Fielding [19], share a similar goal with WS-* web services, which is to increase interoperability for a looser coupling between the parts of distributed applications [20]. However, the goal of REST is to achieve this in a more lightweight and simpler manner seamlessly integrated to the web. REST uses URIs for encapsulating and identifying services on the web. In its web implementation it also uses HTTP as a true application protocol. This way, REST brings services "into the browser": Resources can be linked, bookmarked, cached, searched for, and the results are directly visible within any web browser. Requests for services (i.e., verbs on resources) are formulated using a standard URI. For instance, typing a URI such as http://.../spot1/sensors/temperature in a browser, can be used to request the resource (here: operation) "temperature" of the resource "sensor" of "spot1" with the verb GET HTTP method.

Traditionally, REST has been used to integrate websites together. However, the lightweight and ubiquitous aspects of REST makes it an ideal candidate to build an "universal" API (Application Programming Interface) for embedded devices. This concept is often referred to as "Web of Things" [17], [18], [25].

Since many such devices usually offer rather simple and atomic functionalities (for example reading sensor values), modeling them using REST is often straightforward. While REST services are well adapted for rather atomic services, thus, cover a fair part of the basic services offered by embedded devices, they have limitations when modeling services which require complex input and/or deliver complex outputs. According to our own experience and of others [26], in traditional integration patterns based on WS-* web services, we suggest that WS-* services are to be preferred for highly complex real-world integration and rather static usecases, such as those involving complex business processes or those requiring high reliability or security, for example 


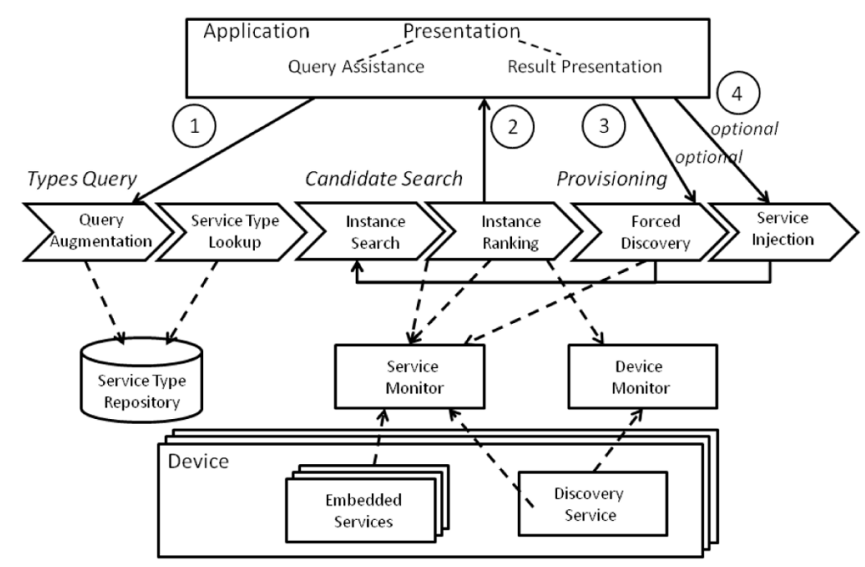

Fig. 4. Overview of the real-world service discovery and provisioning process (RSDPP).

composing a manufacturing process on several machines. For lightweight and more end-user oriented applications, the RESTful approach offers significant advantages such as simplicity, direct web integration, and looser-coupling [4], [26]. As both scenarios are needed for truly flexible enterprise applications, the Local Discovery Unit of our solution supports both DPWS and REST-enabled devices.

HTTP has been designed as a high-level application protocol; therefore, the notion of network discovery is not part of HTTP specifications. In the modern web, resources are discovered by following outgoing links from each resource, but this model requires to know beforehand the URI of a boot-strap resource, therefore, is not suited for discovering new devices that appear on the network. To counter that, mRDP [27] proposed as a simple HTTP-based semantic resource discovery mechanism, based on UDP broadcasting. In our approach, we implemented alongside WS-Discovery, a RESTful network discovery mechanism suited for devices that do not support DPWS, but only HTTP. In the passive network discovery of REST-enabled devices, each device must announce itself using a HELLO message (exactly as in the DPWS network discovery case), however, upon reception of the acknowledgment from the LDU, the device will generate a PUT request on the LDU REST server, and register itself into the LDU using a predefined device registration procedure.

Since there is no standard for the discovery of RESTful services, we cannot expect any RESTful device to be compliant with the custom HTTP passive discovery protocol we have just described. In this case, we force the network discovery (active) upon the device, by issuing a GET request on the device page which needs to be known. The LDU will parse the device page and retrieve the necessary information from the device and its services. The device page is a machine-readable "RESTful API" described in HTML. To enhance this description we experimented using metadata contained in a specific microformat ${ }^{1}$ we have developed specifically for devices. The metadata contained in this microformat is the same as the metadata used in the network discovery process of DPWS devices so that the same information is contained in the service repositories of the architecture for both REST and DPWS services.

1. www.microformats.org.

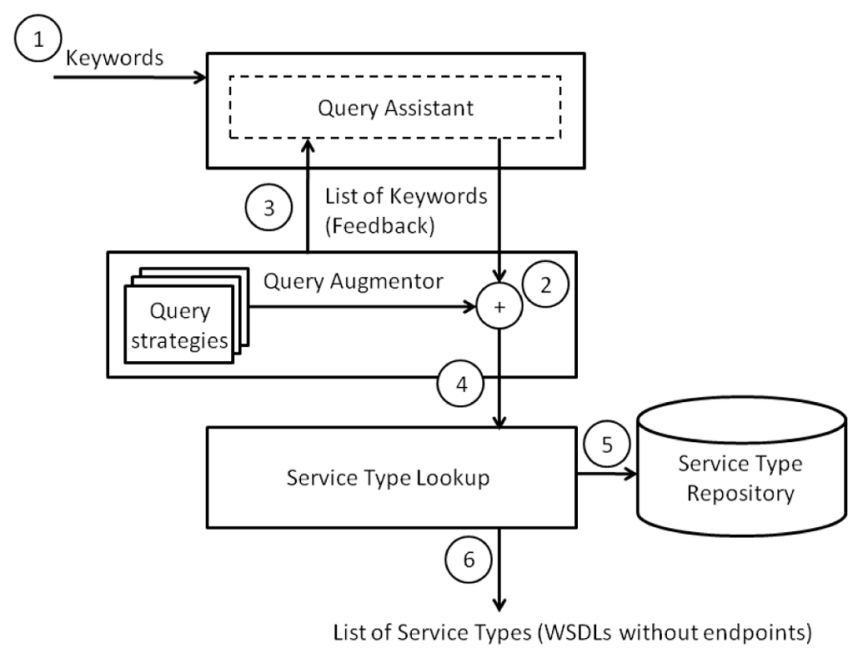

Fig. 5. Looking for a service type.

\section{Real-World Service Discovery and Provisioning Process (RSDPP)}

After describing the way devices and their services are advertised, this Section describes the RSDPP and its underlying steps. As illustrated in step 1 in Fig. 4, the process begins with a Types Query after the network discovery of devices has been executed. In this subprocess, the developer uses keywords to search for services as she would search for documents on any search engine. Subsequently, this query is extended with related keywords fetched from different websites, and used to retrieve types of services that describe the functionality, but not yet the real-world device it runs on. This is the task of the Candidate Search, where the running instances of the service type are retrieved and ranked according to context parameters provided by the developer (step 2, Fig. 4). In case no service instance has been found, the process goes on with Provisioning. It begins with a forced network discovery of devices, where the devices known to provide the service type the developer is looking for, are asked to acknowledge their presence (step 3). If no suitable device is discovered, a service injection can be requested. In this last step the system tries to find suitable devices that could run the requested service, and installs it remotely (step 4).

\subsection{Types Query}

In the first part of the discovery process (step 1 in Fig. 4), the developer or process designer enters keywords describing the type of service she wants to find (step 1 in Fig. 5). A Service Type is a generic WSDL file describing the abstract functionalities of a real-world service, but not bound to any particular end-point of a concrete real-world device. The entered keywords are sent to the Query Augmentation module which extends the query with additional keywords. The output of this module is then used to retrieve and rank types of services according to user-quality parameters such as the current user context.

\subsubsection{Query Augmentation and Assistant}

In conventional service discovery applications, the keywords entered by the user would be sent to a Service Repository to find types of services corresponding to the keywords. The 


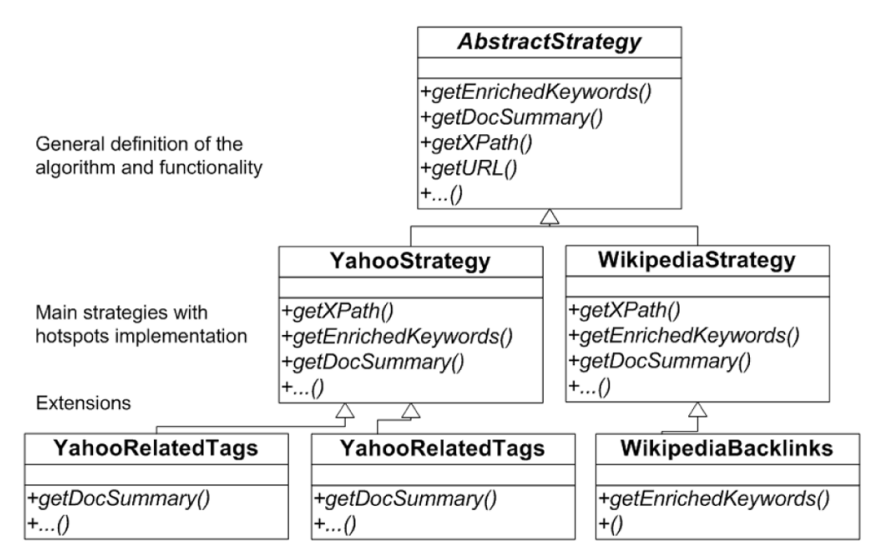

Fig. 6. Architectural overview of the Query Strategies based on the Strategy and Template software design patterns.

problem with this simple keyword matching mechanism is that it lacks flexibility. As an example lets assume a developer who wants to find services offered by a "smart meter", a term often used to describe a next generation device that can measure the energy consumption of other devices and possibly control them depending on built-in logic. Typing "smart meter" only, will likely not lead into finding all the corresponding services, because services dealing with energy consumption monitoring might not be tagged with the "smart meter" keyword but simply with "electronic meter", etc. We want to avoid the construction of domain ontologies, and to minimize the amount of data that embedded devices need to provide upon network discovery and service registration. Thus, we propose a system that uses services on the web to extend queries without involving communication with the embedded devices or requiring complex service descriptions from them. This is the query augmentation shown on step 2 in Fig. 5.

Our idea is to use existing knowledge repositories such as web encyclopedias (e.g., Wikipedia) and search engines (e.g., Google, Yahoo! Web Search), in order to extract "lightweight ontologies" [21] or vocabularies of terms from their semistructured results. The basic concept of the query augmentation (step 2 in Fig. 5) is to call $1 . . n$ web search engines or encyclopedias with the search terms provided by the developer, for instance "smart meter". The XHTML result page from each web resource is then automatically downloaded and analyzed. The result is a list of keywords, which frequently appeared on pages related to "smart meter". A number of the resulting keywords, are thus, related to the initial keyword, i.e., "smart meter", and therefore, can be used when searching for types of services corresponding to the initial input.

An invocable web-resource together with several filters and analysis applied to the results is called a Query Strategy. The structure is based on the Strategy Pattern [28], which enables us to encapsulate algorithms into entirely independent and interchangeable classes. This eases the implementation of new strategies based on web resources containing relevant terms for a particular domain. A simplified class diagram of the Query Strategy framework is depicted on Fig. 6. Any Query Strategy has to implement the AbstractStrategy class which provides the general definition of the algorithm. As an example the YahooStrategy is a concrete implementation of this algorithm using the Yahoo! Search service. Furthermore, strategies can have extensions, adding more specific functionality to a concrete instance of a Query Strategy. As an example, the Wikipediastrategy can be extended with the WikipediaBacklinks class. This particular extension is using the backlinks operation offered by Wikipedia in order to know what pages are linking to the currently analyzed page similarly to what the well-known PageRank uses to rank websites [29]. This information is then used by the WikipediaStrategy to browse to related pages and gather relevant keywords. As such, our approach builds on top of existing ranking and connectivity approaches on the web.

Furthermore, Query Strategies can be combined in order to get a final result that reflects the successive results of calling a number of web-resources. The resulting list of related keywords is then returned to the developer in the Query Assistant, where she can (optionally) remove keywords that are not relevant (step 3 in Fig. 5).

The implementation of the Query Strategy architecture makes it easy to test combinations of several strategies together with their extension. We implemented a number of these, and their evaluation is presented in Section 5.2.

\subsubsection{Service Type Lookup}

The augmented query is used to determine any matching service types in the Service Type Repository (step 4 and step 5 in Fig. 5). All service types that match any of the keywords supplied are found; both those manually entered and those determined automatically by the augmentation step. The query keywords are matched against all metadata of a service type that was sent to the Service Monitor upon network discovery or extended by a manual entry. This includes human readable descriptions, contact information, legal terms, explicit keywords, and interface descriptions (WSDL). Additionally, structured technical metadata is considered, e.g., dependency information between service types, and requirements of the service type on underlying hardware. The result of the Service Type Lookup is a list of service types that potentially support the functionality the developer is looking for.

\subsection{Candidate Search}

Real-world devices are volatile, e.g., often connecting and disconnecting, thus, we need to decouple the discovery of service types from the discovery of actual instances of services. The Candidate Search (step 2 in Fig. 4) models the discovery of running service instances. The first step in this subprocess is for the developer to select the suitable types of services by browsing their details (step 1 in Fig. 7). Alternatively she can select all the types retrieved in the Types Query part of the process.

\subsubsection{Context Extractor}

One of the main differences between real-world service and virtual services is that real-world services are directly linked to the physical world. As a consequence, the context in which a service exists as well as the context in which the developer initiates the discovery of a service are highly relevant. Context is information that qualifies the physical world, and it can help in both reducing the number of 


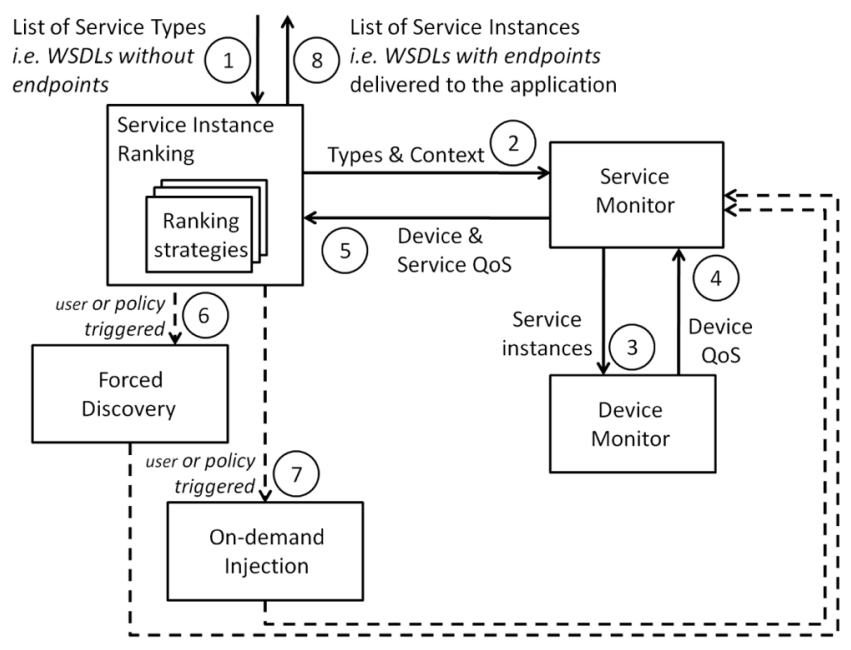

Fig. 7. Ranking and optionally Provisioning Service Instances.

services returned to the developer, as well as in finding the most appropriate services for the current environment [30].

To satisfy the requirements of real-world service discovery, we propose modeling the context by two distinct parts inspired from [31]: the digital environment, which we define as everything that is related to the virtual world the developer is using, and the physical environment, which refers to properties of the physical situation the developer currently is located in or wants to discover services about.

The digital environment is composed of Application Context and Quality of Service. The Application Context describes the business application the developer uses when trying to discover services, e.g., the type of application she is currently developing or the language currently set as default. Such information codetermines the services a developer is looking for and can reduce the discovery scope. The QoS Information reflects the expectations of the developer (or of the application she is currently using) in terms of how the discovered service is expected to perform. Our current implementation supports service health and network latency, i.e., the current status of the service and the network transmission delay usually measured when calling it.

The physical environment is mainly composed of information about location. Developers are likely to be looking for real-world services located at a particular place, unlike when searching most virtual services. We decompose the location into two subparts following the Location API for Mobile Devices (as defined in Java Specification Request JSR-179). The Address encapsulates the virtual description of the current location, with information such as building name, floor, street, country, etc., and the Coordinates are GPS coordinates. In our implementation the location can either be automatically extracted, e.g., if the developer looks for a real-world service close to her location, or it can be explicitly specified if she wants a service located close to a particular location, e.g., in a form of radius.

In the RSDPP Context, extraction on the developer side is done at step 2 in Fig. 7. It is worth noting that the context on the developer side is meant to reflect the expectations or requirements with regard to the services that are going to be returned. As an example, during this phase the developer can express the wish for a service to be physically close to her current location, or she can quantify the importance of context parameters such as Quality of Service.

This developer-quality information is then going to be compared to the service and device side context by the Service Instance Ranking component (see Section 4.2.3) in order to select and rank the most relevant service instances.

\subsubsection{Service Instance Search}

In step 3 in Fig. 7, the identifiers of the selected service types and the context object extracted on the developer-side are sent to the Service Monitor. This component is the link between service types and running instances of these services. Thanks to the dynamic network discovery of devices (explained in Section 3.1) the Service Monitor and the Device Monitor know what devices are currently providing which service types. In steps 4 and 5 in Fig. 7, the Service Monitor queries the Device Monitor for the context of the selected service instances. The digital environment context parameters such as the Quality of Service, are derived by polling the devices from time to time, as well as by monitoring the invocations of services and calculating their execution time.

Getting the context parameters related to the physical environment of a service instance is slightly more complicated. Indeed, as an example it can not be expected from each real-world device to know its location. Thus, we suggest taking a best effort strategy, where each actor of the discovery process is trying to further fill-in the context object. As an example, consider a mobile sensor node without a coordinates-resolving module (e.g., a GPS). When discovered by the Local Discovery Unit (see Section 3.1 and Fig. 2), the sensor node does not know its location, and thus, can not fill-in the Address and Coordinates fields of the context object. The LDU however, is a usually immobile component and can be configured at setup time with its location and current address. As a consequence the LDU can fill the Address and Coordinate fields of the sensor node with its own location (within a specific radius). While not entirely accurate with respect to the sensor's exact location, this information will already provide a hint which can be of value to the developer. In the future, more sophisticated methods can be used at device or LDU level (especially if any of them are mobile), e.g., [32] in order to acquire location information with the required accuracy. Similarly, since we can not expect every LDU to provide a full contextual profile, the Service Monitor has its own default context component which can again be used to extend the information the device and LDU provided. The final context information is packed into a context object for each device running the selected Service Instances.

If no appropriate service instances have been found, the optional step 7 (described in Section 4.3) and step 8 (described in Section 4.3.2) are taken; otherwise, the process continues with Service Instance Ranking.

\subsubsection{Service Instance Ranking}

The Service Instance Ranking component is responsible for sorting the instances according to their compliance with the context specified by the developer or extracted from her machine. As shown in step 6 in Fig. 7, the Service Instance Ranking component receives a number of service instances alongside with their context object. It then uses a Ranking Strategy to sort the list of instances found. For instance, a 
Ranking Strategy could use the network latency so that the services are listed sorted according to their network latency; another could rank instances according to their compliance with the current location of a developer or the target location she provided.

As for Query Strategies (see Section 4.1.1), Ranking strategies can be well modeled using the Strategy pattern. In this way, new strategies can be easily implemented and integrated. Furthermore, we extend the pattern to support chained ranking strategies, in order for the resulting ranking to reflect a multicriteria evaluation. Each ranking criterion can use both the context information of the instances gathered during the Service Instance Search, and the context information extracted on the developer side in step 2 in Fig. 7. Thus, instances can be ranked against each other and/or against the context of the developer (e.g., her location). The output of the ranking process is an ordered list of running service instances corresponding both to the extended keywords and to the requirements in terms of context expressed by the developer. The pattern-based design of the component makes it possible to extend the strategies to accommodate emerging research, e.g., [33], [34], [35] on matching and ranking that needs, however, to be adapted to consider the specifics of real-world services.

\subsection{On-Demand Service Provisioning}

In case no running service instance has been found, OnDemand Service Provisioning will first actively try to discover service instance on the network that matches the developer's requirements. If this also fails, installation of services on suitable devices will be carried out.

\subsubsection{Forced Network Discovery of Devices}

As discussed in Section 3.1, passive DPWS network discovery can be unreliable depending on the mechanism used. This is due to the fact that UDP is used, which provides an unreliable service where datagrams may arrive out of order, appear duplicated, or simply get lost without notification. Furthermore, this mechanism might take a long time to propagate across the whole system (especially when we have thousands of on-device services, e.g., [36]) as UDP packets are multicasted only in local networks. When up-todate information is needed, the forced network discovery mechanism can be used, particularly within dynamic environments where devices with unknown capabilities continuously connect to or disconnect from the network. This dynamic process can use different types of filters to specify the device type and the scope, as well as other additional semantic information. This is useful to restrict the result set when looking for new devices, as only devices matching the specified criteria will respond. Forced network discovery is depicted as the optional step 6 in Fig. 7. Forced discovery will result to new discovery messages arriving at System Monitor, after which the process is continued from step 5.

\subsubsection{On-Demand Service Injection on Devices}

In case that even after forced network discovery of devices no service instances that match the query have been identified, the system tries to inject (i.e., remotely install) appropriate instances of the identified service types (depicted as the optional step 7 in Fig. 7). This involves finding devices that are capable of hosting the service, and actually installing it in a platform-dependent way. The new instances are detected by the System Monitor, and again, the process continues from step 5 .

Injection is possible if the descriptions of the service types identified in previous steps include installation instructions and executable software artifacts. In the Service Repository, for each service type, we, therefore, provide data structures for deployable artifacts, including (dynamic and static) hardware requirements and dependency relations between services. These requirements are compared with the capabilities and states of the currently available devices. An efficient service to device mapping is calculated and platform-specific injection actions are taken to change the system according to the mapping. Once the injection finishes successfully, control is handed back to Service Instance Ranking, which uses the Service Monitor again to discover the newly installed Service Instances.

In a concrete example, the service description of a fire detection service could include both DPWS bundle for installation on an DPWS-enabled sensor platform and a rule set for a rule-based sensing system. Meta information makes sure that the bundle and the rule set are only applied to the appropriate platforms. Additional information for deployment can be included, such as the desired coverage (e.g., 80 percent of all nodes), dependency on other services, e.g., a temperature measurement service and a fire shutter control service. If the service to be deployed depends on other services, those can be deployed as well. Metadata may also include the memory required to install the new service. Our efforts on service mapping and injection are described in detail in [22].

\section{Process Evaluation}

A prototype of the described process was implemented and integrated to the SOCRADES Integration Architecture. The prototype implementation was developed in Java and deployed on a Java Enterprise Application Server (SAP NetWeaver) at two distinct locations. The evaluation of the prototype was split in three parts following the subparts of the Real-World Service Discovery Process (i.e., Types Query, Candidate Search, Provisioning).

The first step in evaluating the implementation of the process was to get a number of DPWS-enabled devices offering services to search for. Unfortunately, since it is only recently that DPWS has become an official standard (WS-DD), its adoption on industrial devices is ongoing. Thus, we decided to simulate a larger number of devices that one could expect to find in future industrial environments. Since developers usually write the description of web services [11], we selected 17 experienced developers and asked them to write the description of a selected device and of at least two services it could offer. The developers were given the documentation of a concrete device according to the projects they were currently working on. Based on these descriptions we generated 30 types of services (described in WSDL containing DPWS metadata) for 16 different smart devices ranging from RFID readers to robots and sensor boards. Out of these, 1,000 service instances were simulated at the two deployed locations.

This work further extended the simulation by implementing them on a prototyped shop floor in a laboratory and industrial setup in two different scenarios described in 


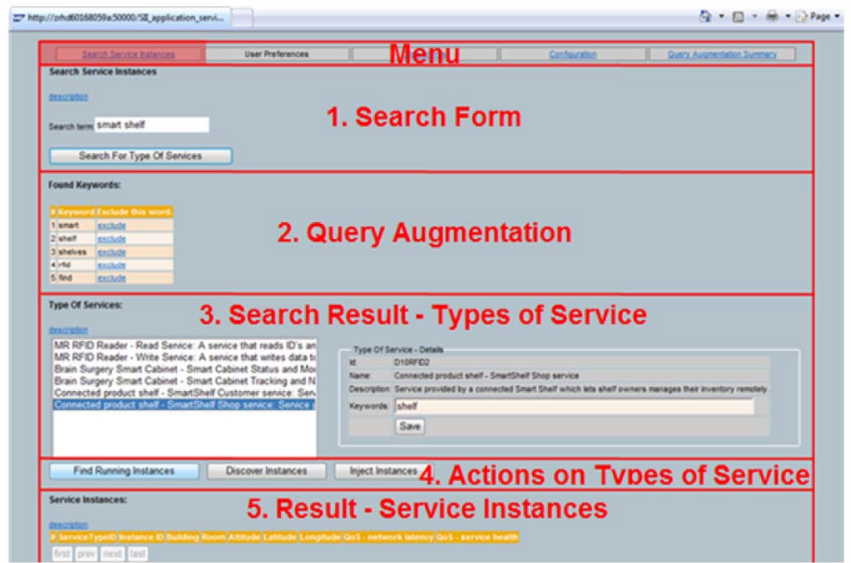

Fig. 8. Search user interface, looking for instances of services in the application service catalogue.

Section 5.4. The locations spanned across cities and countries using Internet as the communication backbone for these services and internal operations. Common shop floor devices like temperature and vibration sensors were identified. SunSPOT sensors, ${ }^{2}$ gantry robots, PLC (Programmable Logic Controller) devices controlling conveyor belts and proximity sensors from leading industrial vendors were wrapped with (DPWS) web services or directly deployed service instances on the devices themselves. We further tested the integration of RESTful devices by implementing a native web server for the SunSPOTs [18].

The prototype of the SOCRADES Integration Architecture was used in the back end to monitor, search and compose services offered by these devices. The LDU of the SIA was used at different locations in the field trials to dynamically discover devices on the shop floor.

\subsection{Search User Interface}

A search (or discovery) web user interface (UI) for developers was developed (shown on Fig. 8) on the top of the Java Enterprise implementation of the RSDDP. This UI offers several interaction zones corresponding to the steps of the process and allows the developer to feed her feedback into the discovery loop at every step of the process.

In the first zone (1. Search Form) the developer is asked to enter $n$ keywords. These keywords are then extended with related words by the Query Augmentation and Assistant module (see Section 4.1.1 and step 1 in Fig. 4), as shown in the second zone (2. Query Augmentation). In this zone, the developer can also choose to remove/extend words before performing a Service Type Lookup (second part of step 1 and Section 4.1.2). The result of this is shown in the third zone (3. Search Result-Types of Services) that is a list of all the types of services corresponding to the extended keywords. The developer then selects the types she is actually interested in. To help her identifying these, a table provides a description of each type as well as all its currently associated tags. Additionally, the developer can tag the service types with new keywords, if she thinks relevant tags are still missing. Once the service types are selected, the Candidate Search is performed (step 2 in Fig. 4 and Section 4.2. The result of this execution (zone 5. ResultService Instances) is a list of running instances for all the

2. www.sunspotworld.com.

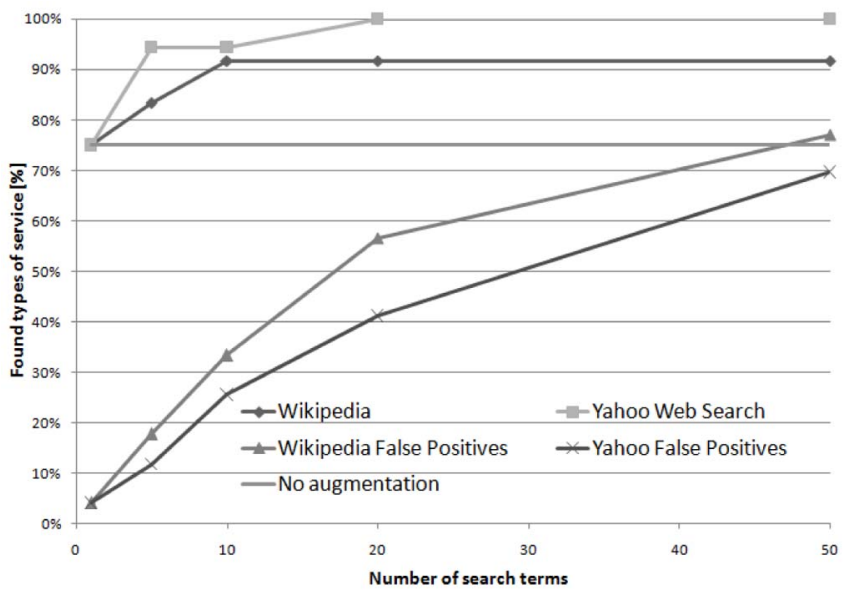

Fig. 9. Results for the Query Augmentation with Yahoo! and Wikipedia

selected types. As already mentioned, to facilitate the final selection of a service, the instances are ranked according to the context parameters extracted on the developer side. As an example, the service instances on Fig. 8 are sorted according three combined parameters, i.e., network latency, service health, and location (with respective relevance levels of 10 percent, 30 percent, and 50 percent).

Before taking the final decision on what service to select the instances can be tested. In the case of a (DP)WS service a click on a instance results in opening its complete WSDL file, and a further click on a link opens the Web Service Navigator of SAP Netweaver to allow testing of the service. In the case of a RESTful service a click on an instance directly retrieves the identifier of the service (i.e., a URL) and allows for direct testing within the browser.

Finally, if no running instances were found for any of the selected types, the developer can choose to force the network discovery of instances (step 3 in Fig. 4 and Section 4.3) or to perform an injection of the selected types on appropriate embedded devices (zone 4. Actions on Types of Service).

\subsection{Evaluation of Types Query and Candidate Search}

In the evaluation of the Query Augmentation module we wanted to know whether:

1. Augmenting developer input with related keywords could help in finding more real-world web services

2. What type of combination of query strategies is the most suitable.

Two types of strategies were used. In the first, we used a human generated index (i.e., Wikipedia), and in the second, a robot generated index (i.e., Yahoo! Web Search). The input keywords were selected by seven volunteers, all working in IT. They provided 71 search terms (composed of one to two words) that they would use if they were to search for services provided by the 17 devices. These terms were entered one by one and all the results were logged.

The trend extracted from these experiments is shown on Fig. 9. Two results can be drawn. First the Query Augmentation process does indeed help in finding more real-world services. Without augmentation 75 percent (plain gray line on Fig. 9) of the service types were found and using the query augmentation up to a 100 percent. However, the Query Augmentation generates a number of false positives, 
i.e., service types that are returned even if they are not related to the provided keywords (depicted by the two lines at the bottom of Fig. 9). Thus, we need to restrict the number of keywords added to the initial ones. The observed optimum is between 5 and 10 added keywords, leading to less than 20 percent false positives out of 95 percent types of services found. The second result can be seen on Fig. 9 which reveals that using Yahoo!, the approach performs slightly better than when using Wikipedia. Looking more in detail, we see that indeed, approximately 50 percent of the keywords used against Wikipedia did not lead to any page, simply because they do not have yet dedicated articles, even if Wikipedia was growing at a rate of about 1,400 articles per day. ${ }^{3}$ However, when results where extracted from Wikipedia pages they were actually more relevant for the searched real-world services. Thus, a good solution would be to chain the strategies so that first human generated indexes are called and then robot generated ones, in case the first part did not lead to results.

The Candidate Search was evaluated based on a proof of concept implementation. We tested two chained ranking strategies for the generated services: one comparing service health and given weight of 30 percent as well as one comparing network latency and given a weight of 50 percent. They performed as expected, sorting the lists of retrieved service instances according to the ranking strategies which, we believe helps developers finding their way across the results, but would need to be tested with neutral volunteers. We implemented the sorting using the merge sort algorithm which has a complexity of $O(n \log n)$, and since the strategies can be chained we have an overhead for the ranking of $O(m n \log n)$ where $m$ is the number of strategies and $n$ the number of Service Instances.

\subsection{Evaluation of On-Demand Service Provisioning}

On-Demand Service Provisioning can be the result of not being able to find a suitable running service, or even because the possible existence of such a service on a specific device would result in better satisfying the requirements, e.g., better performance. Our implementation of the on-demand provisioning part was done using adaptations of well-known algorithms, which resulted to NP-hard approaches for service to device mapping [22]. Both probabilistic/efficient $(O(n k))$ and complete/inefficient $\left(O\left(n^{k}\right)\right)$ algorithms have been implemented. Some evaluation was done using test scenarios, in which the probabilistic algorithms produced results close to the optimum, with respect to a given objective function. A proof of concept implementation demonstrated the service mapping and deployment both on simulated and real devices (PDA-level). Flexibility is achieved by using exchangeable strategies for each step of the mapping process that can be exchanged at during runtime. This approach is also scalable, since most of the components can be easily replicated and distributed across different locations. A detailed evaluation and discussion of the on-demand service provisioning is given in [22].

\subsection{Demonstrator and Field Trial}

In the recent years we have witnessed the SOA concepts starting to be successfully applied to the shop floors of future factories. Thus, the key motivation for evaluating the

3. http://en.wikipedia.org/wiki/Wikipedia:Size_of_Wikipedia.

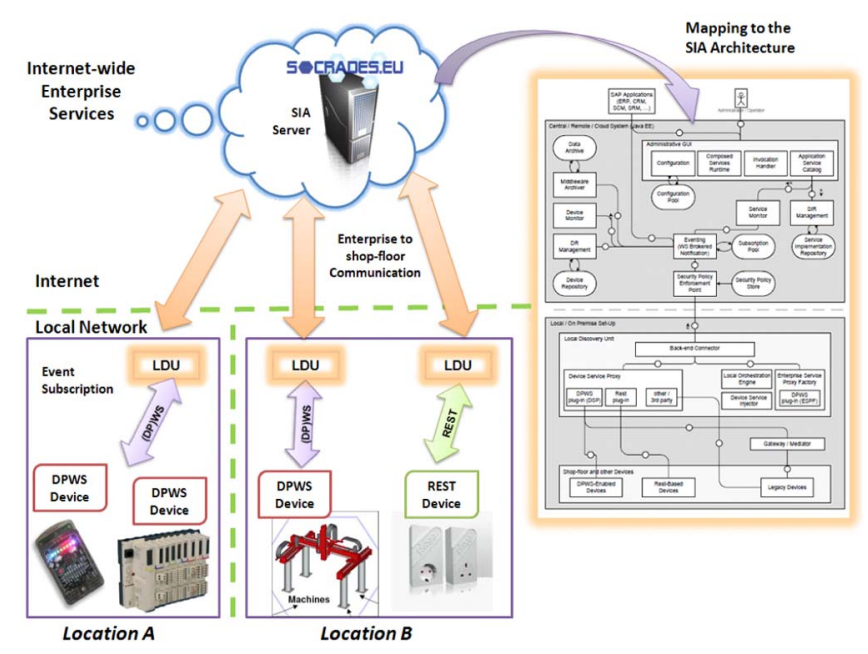

Fig. 10. Trial of the SIA in a multilocation, cross-network real-world scenario.

prototype was to identify the feasibility of SOA based cross-location infrastructure in real-world situations such as in factories. In the demonstrated scenario multiple heterogeneous physical devices on the shop floor were discovered, the services to be composed were identified and used based on the RSDPP. In particular, DPWS and RESTful web services were deployed (see Fig. 10) with the aim to evaluate part of the aforementioned concepts on real data and events.

In the trial, a manufacturer operates in two distinct geographical locations (marked as "Location $\mathrm{A}^{\text {" and }}$ "Location B" in Fig. 10), that also belong to different networks and reside behind firewall/proxy without direct connections between the shop-floors. At company's headquarters a running ERP system governs the overall operations with respect to the two locations. A production order is issued from the headquarters to its production facilities at location A. During the course of production, a severe unpredictable failure of machines causes the production to be stopped at location A. Such a scenario occurs often in production plants where the stop per hour could run to a loss of thousands of dollars.

In this scenario the ERP system at the headquarters is immediately alerted about the current loss and an estimated delay in completing the production order is calculated. Subsequently the ERP evaluates alternative scenarios in order to realize the customer's order and satisfy its constraints. Therefore, it decides to relocate the remaining of the production order to location B. It also arranges for the already produced parts of location $\mathrm{A}$ to be transferred to a storage room where the parts from location B will also arrive shortly, in order to complete one whole shipment to the customer.

During the design time of the composite application, the services hosted on the devices at these facilities are discovered by the SIA and its LDUs. These services, along with their QoS and other contextual information, are stored in the Service Type Repository. A sequential processoriented composite application is created by the developer by searching for instances of services using the RSDPP and the search user interface in order to complete a production sequence. Functionality envisioned at design time but that could not be found at runtime is deployed on-demand. This 
composite application is then also available as a web service. Two such applications were developed for location A and location B for this scenario. In the second phase-runtime phase, the actual composite application is being executed.

\section{Conclusion}

The future Internet will be highly populated by heterogeneous networked embedded devices that will further blur the borders of real and virtual world, empowering us with new capabilities in creating real-world aware business applications. For this to happen, it is of high importance to be able to find real-world services that can be dynamically included in enterprise applications-a quite challenging task considering the application requirements, technologies, and heterogeneity of devices. In that line of thought, we have presented here an approach that would facilitate this task for developers, allowing them not only to search efficiently for services running on embedded devices, but also to deploy missing functionalities on-demand.

The comprehensive process demonstrated in this paper shows that we can extend the reach of enterprise computing to the real world (and vice versa). To achieve this, we suggest to use web service standards (DPWS) and web-oriented patterns (REST) to easily integrate physical devices into existing enterprise information systems. Web services on devices can be used to dynamically register devices and the service(s) they provide. We have suggested to use queries to search service metadata that has been gathered by the network discovery of devices. Furthermore, we have designed and evaluated automatic augmentation of the search queries, with strategies that extend queries with related keywords found on knowledge repositories available on the network, e.g., third party websites. With this extension we have shown that significantly more services can be identified without overloading devices with description data. We have also shown how context is important for real-world services and explained its use within the service discovery process. Finally, we presented how missing functionality can be injected on devices upon developer's request.

\section{ACKNOWLEDGMENTS}

The authors would like to thank the European Commission and the partners of the SOCRADES (www.socrades.eu) project for their support.

\section{REFERENCES}

[1] E. Fleisch and F. Mattern, Das Internet der Dinge: Ubiquitous Computing und RFID in der Praxis:Visionen, Technologien, Anwendungen, Handlungsanleitungen. Springer-Verlag, 2005.

[2] D. Lizcano, M. Jiménez, J. Soriano, J.M. Cantera, M. Reyes, J.J. Hierro, F. Garijo, and N. Tsouroulas, "Leveraging the Upcoming Internet of Services Through an Open User-Service Front-End Framework," Proc. First European Conf. Towards a Service-Based Internet (ServiceWave '08), pp. 147-158, 2008.

[3] P.J. Marrón, S. Karnouskos, and D. Minder, Research Roadmap on Cooperating Objects. European Commission, Office for Official Publications of the European Communities, July 2009.

[4] D. Guinard and V. Trifa, "Towards the Web of Things: Web Mashups for Embedded Devices," Proc. Workshop Mashups, Enterprise Mashups and Lightweight Composition on the Web (MEM '09), Apr. 2009.
[5] M. Hatler, D. Gurganious, C. Chi, and M. Ritter, "WSN for Smart Industries," OnWorld Study, www.onworld.com, 2007.

[6] M. Marin-Perianu, N. Meratnia, P. Havinga, L. de Souza, J. Muller, P. Spiess, S. Haller, T. Riedel, C. Decker, and G. Stromberg, "Decentralized Enterprise Systems: A Multiplatform Wireless Sensor Network Approach," IEEE Wireless Comm., vol. 14, no. 6, pp. 57-66, Dec. 2007.

[7] W.K. Edwards, "Discovery Systems in Ubiquitous Computing," IEEE Pervasive Computing, vol. 5, no. 2, pp. 70-77, Apr./Jun. 2006.

[8] F. Jammes and H. Smit, "Service-Oriented Paradigms in Industrial Automation," IEEE Trans. Industrial Informatics, vol. 1, no. 1, pp. 62-70, Feb. 2005.

[9] L.M.S. de Souza, P. Spiess, D. Guinard, M. Köhler, S. Karnouskos, and D. Savio, "SOCRADES: A Web Service Based Shop Floor Integration Infrastructure," Proc. Internet of Things Conf. (IoT '08), pp. 50-67, Mar. 2008.

[10] N.B. Priyantha, A. Kansal, M. Goraczko, and F. Zhao, "Tiny Web Services: Design and Implementation of Interoperable and Evolvable Sensor Networks," Proc. Sixth ACM Conf. Embedded Network Sensor Systems, pp. 253-266, 2008.

[11] M. Crasso, A. Zunino, and M. Campo, "Easy Web Service Discovery: A Query-by-Example Approach," Science of Computer Programming, vol. 71, no. 2, pp. 144-164, Apr. 2008.

[12] C. Atkinson, P. Bostan, O. Hummel, and D. Stoll, "A Practical Approach to Web Service Discovery and Retrieval," Proc. Int'l Conf. Web Services (ICWS '07), pp. 241-248, 2007.

[13] R. Monson-Haefel, J2EE Web Services: XML SOAP WSDL UDDI WS I JAX-RPC JAXR SAAJ JAXP. Addison-Wesley Professional, Oct. 2003.

[14] H. Song, D. Cheng, A. Messer, and S. Kalasapur, "Web Service Discovery Using General-Purpose Search Engines," Proc. IEEE Int'l Conf. Web Services (ICWS '07) , pp. 265-271, 2007.

[15] W. Drytkiewicz, I. Radusch, S. Arbanowski, and R. PopescuZeletin, "pREST: A REST-Based Protocol for Pervasive Systems," Proc. IEEE Int'l Conf. Mobile Ad-Hoc and Sensor Systems, pp. 340348, 2004.

[16] D. Guinard, V. Trifa, P. Spiess, B. Dober, and S. Karnouskos, "Discovery and On-Demand Provisioning of Real-World Web Services," Proc. IEEE Int'l Conf. Web Services (ICWS '09), pp. 583590, 2009.

[17] E. Wilde, "Putting Things to REST," Technical Report UCB iSchool Report 2007-015, School of Information, Univ. of California, Berkeley, Nov. 2007.

[18] D. Guinard, V. Trifa, T. Pham, and O. Liechti, "Towards Physical Mashups in the Web of Things," Proc. IEEE Sixth Int'l Conf. Networked Sensing Systems (INSS '09), pp. 196-199, June 2009.

[19] R.T. Fielding and R.N. Taylor, "Principled Design of the Modern Web Architecture," ACM Trans. Internet Technology, vol. 2, no. 2, pp. 115-150, 2002.

[20] C. Pautasso and E. Wilde, "Why is the Web Loosely Coupled? A Multi-Faceted Metric for Service Design," Proc. 18th Int'l World Wide Web Conf. (WWW'09), http://www.jopera.org/node/190, Apr. 2009.

[21] M. Hepp, K. Siorpaes, and D. Bachlechner, "Harvesting Wiki Consensus: Using Wikipedia Entries as Vocabulary for Knowledge Management," IEEE Internet Computing, vol. 11, no. 5, pp. 5465, Sept./Oct. 2007.

[22] T. Frenken, P. Spiess, and J. Anke, "A Flexible and Extensible Architecture for Device-Level Service Deployment," Proc. First European Conf. Towards a Service-Based Internet (ServiceWave '08), pp. 230-241, 2008.

[23] S. Karnouskos, O. Baecker, L.M.S. de Souza, and P. Spiess, "Integration of SOA-Ready Networked Embedded Devices in Enterprise Systems via a Cross-Layered Web Service Infrastructure," Proc. IEEE Conf. Emerging Technologies \& Factory Automation (ETFA), pp. 293-300, Sept. 2007.

[24] P. Spiess, S. Karnouskos, D. Guinard, D. Savio, O. Baecker, L.M.S. d. Souza, and V. Trifa, "SOA-Based Integration of the Internet of Things in Enterprise Services," Proc. IEEE Int'l Conf. Web Services (ICWS '09), pp. 968-975, 2009.

[25] T. Luckenbach, P. Gober, S. Arbanowski, A. Kotsopoulos, and K. Kim, "TinyREST-A Protocol for Integrating Sensor Networks into the Internet," Proc. Workshop REALWSN, 2005.

[26] C. Pautasso, O. Zimmermann, and F. Leymann, "RESTful Web Services versus 'Big' Web Services: Making the Right Architectural Decision," Proc. 17th Int'l World Wide Web Conf. (WWW '08), 2008. 
[27] J.I. Vazquez and D.L. de Ipina, "mRDP: An HTTP-Based Lightweight Semantic Discovery Protocol," Computer Networks J., special issue on innovations in web comm. infrastructure, vol. 51, no. 16, pp. 4529-4542, 2007.

[28] E. Gamma, R. Helm, R. Johnson, and J.M. Vlissides, Design Patterns: Elements of Reusable Object-Oriented Software. AddisonWesley Professional, Mar. 1995.

[29] S. Brin and L. Page, "The Anatomy of a Large-Scale Hypertextual Web Search Engine," Computer Networks and ISDN Systems, vol. 30, nos. 1-7, pp. 107-117, 1998

[30] W. Balke and M. Wagner, "Through Different Eyes: Assessing Multiple Conceptual Views for Querying Web Services," Proc. 13th Int'l World Wide Web Conf., pp. 196-205, 2004.

[31] A. Schmidt, M. Beigl, and H. Gellersen, "There is More to Context than Location," Proc. Int'l Workshop Interactive Applications of Mobile Computing (IMC '98), vol. 23, pp. 893-901, Nov. 1998.

[32] R. Weizheng, "A Rapid Acquisition Algorithm of WSN-Aided GPS Location," Proc. Second Int'l Symp. Intelligent Information Technology and Security Informatics (IITSI '09), pp. 42-46, Jan. 2009.

[33] A. Segev and E. Toch, "Context-Based Matching and Ranking of Web Services for Composition," IEEE Trans. Services Computing, vol. 99, no. 1, pp. 210-222, Jun. 2009.

[34] H. Chan, T. Chieu, and T. Kwok, "Autonomic Ranking and Selection of Web Services by Using Single Value Decomposition Technique," Proc. IEEE Int'l Conf. Web Services (ICWS '08), pp. 661666, Sept. 2008.

[35] V.X. Tran and H. Tsuji, "QoS Based Ranking for Web Services: Fuzzy Approaches," Proc. Fourth Int'l Conf. Next Generation Web Services Practices (NWESP '08), pp. 77-82, Oct. 2008.

[36] S. Karnouskos and A. Izmaylova, "Simulation of Web Service Enabled Smart Meters in an Event-Based Infrastructure," Proc. Seventh IEEE Int'l Conf. Industrial Informatics (INDIN '09), pp. 125130, June 2009.

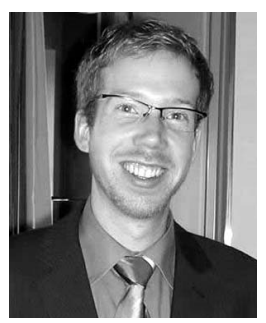

Dominique Guinard received the BSc degree in computer science and management and the MSc degree in computer science from the Universities of Fribourg and Bern. He is working toward the $\mathrm{PhD}$ degree at the Institute for Pervasive Computing at ETH Zurich and is a research associate for SAP Research Zurich. Formerly, he was a researcher at the Auto-ID Labs of ETH working on business mobile interactions with the Internet of Things for Nokia. Before this, he was a scientific collaborator of the software engineering group of the University of Fribourg, where he worked on scalable software architectures for the Internet of Things together with SUN Microsystems Switzerland. His master's thesis was done as a visiting researcher at the Ubicomp Lab of Lancaster University, United Kingdom, with Professor Hans Gellersen, where he worked on using sensor networks to support mobile spontaneous interactions with the physical world. His research interests include web and service-oriented architectures toward a lightweight and composable web of embedded devices. He is a student member of the IEEE.

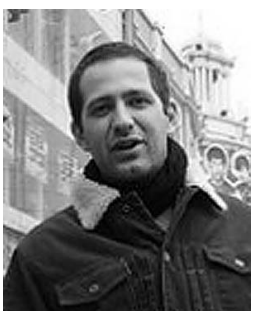

Vlad Trifa received the MSc degree in computer science from the Ecole Polytechnique Fédérale de Lausanne (EPFL) in April 2006. He is working toward the PhD degree in the Institute for Pervasive Computing at ETH Zurich and has been a research associate at SAP Research in Zurich since May 2007. His thesis project was done at the University of California, Los Angeles (UCLA), with Professor Charles Taylor in the Department of Organismic Biology, Ecology, and Evolution, jointly with Professor Deborah Estrin at the Center of Embedded Networked Sensing (CENS), where he developed a framework to use sensor networks to study the influence of sexual and natural selection upon the evolution of bird songs in real environments. Between May 2006 and April 2007, he spent one year as a researcher in the Humanoid Robotics and Computational Neuroscience Laboratories at the ATR Research Center near Kyoto, Japan, where he worked on human-robot interaction using video and audio information. His research interests include lightweight middlewares for embedded devices. He is a student member of the IEEE.

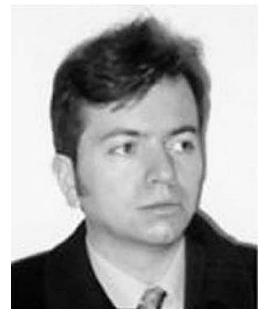

Stamatis Karnouskos is a senior researcher at SAP, investigating the added value of integrating networked embedded devices in enterprise systems. For the last 15 years, he has been involved in more than a dozen European Commission and industrial projects related to Internet-based services and architectures, embedded systems, software agents, mobile commerce, security, and mobility. His research aims at designing and implementing secure open communication infrastructures in enterprise environments. He has authored more than 70 technical papers, acted as guest editor of the IEEE Transactions on Systems, Man, and Cybernetics and Elsevier's Electronic Commerce Research and Applications journals, and participated in the program committees of several international conferences and workshops. He is a senior member of the IEEE.

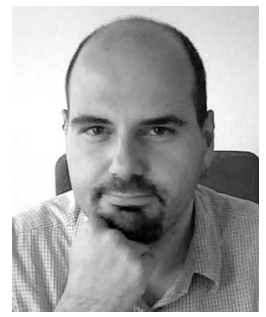

Patrik Spiess received the master's degree in computer science from the University of Karlsruhe, Germany, in 2004. Since then, he has been working for SAP Research as a researcher for different projects in the areas of wireless sensor (actuator) networks, future automation and manufacturing technology, and future energy markets and smart grids. His primary research interests include more closely coupling business IT systems with the physical world, extending their reach and helping businesses to achieve new levels of efficiency and transparency. He is also interested in the fields of ubiquitous computing, service science, and user-friendly and developerfriendly software engineering including end-user development. He is a member of the IEEE.

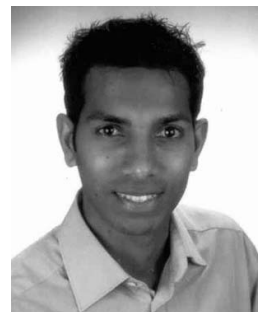

Domnic Savio received the bachelor's degree in electronics and communication engineering and the master's degree in computer science. $\mathrm{He}$ received the PhD degree in computer sciences from the University of Heidelberg, Germany. He joined SAP Research in 2007 at the Campus Engineering Centre in Karlsruhe. Previously, he worked at the Lab for Emerging Technologies in Infienon AG in Munich, Germany, where he did his dissertation on wearable electronics. $\mathrm{He}$ is actively involved in EU-funded projects SOCRADES and the SmartHouse/SmartGrid, where sensors and embedded controllers were interfaced to enterprise computing software. He is also involved in similar activities targeting energy efficiency at the national (German) level with the German Ministry of Technology and Economics (BMWi), e.g., MEREGIO project. He has published more than 15 papers in peer reviewed IEEE conferences and journal publications. He is interested in identifying how smart devices can be integrated to enterprise business processes. $\mathrm{He}$ is a member of the IEEE. 